\title{
Integración curricular: cultura de paz y derechos humanos en Centroamérica
}

Oscar Aristides Alonso Castilla ${ }^{1}$

\section{INFORMACIÓN DEL ARTÍCULO}

Recibido: 30.04 .2020

Aprobado: 30.05 .2020

\section{Palabras claves:}

Cultura de paz,

currículo centroamericano,

derechos humanos.

\section{RESUMEN}

Los altos índices de violencia e inseguridad en la región centroamericana afectan aspectos tanto económicos, como políticos y sociales. Los esfuerzos de integración centroamericana en temas educativos que contemplen la cultura de paz en sus planes curriculares están en un segundo plano; puesto que las prioridades estatales del istmo se han centrado más en temas de cooperación económica - financiera y aduanera.

\section{Curricular integration: culture of peace and human rights in Central America}

\begin{abstract}
The high rates of violence and insecurity in the Central American region affect both economic, political and social aspects. The efforts of Central American integration in educational issues that contemplate the culture of peace in their curricular plans are in the background; since the state priorities of the isthmus have focused more on issues of economic, financial and customs

cooperation.
\end{abstract}

\section{Keywords:}

Culture of peace,

Curriculum,

Human Rights. 


\section{REFLEXIONES EDUCATIVAS}

\section{Introducción}

Lamentablemente, uno de los temas en los cuales la región centroamericana no ha tenido éxito es en el tema de cultura de paz y derechos humanos. Si bien, a finales de siglo pasado, con los acuerdos de Esquipulas I y II, la región centroamericana inicio el rumbo hacia la pasificación del istmo junto a nuevos gobiernos de corte democrático. La actual realidad de la región, en plena globalización, es una realidad complicada; el crimen organizado, la delincuencia y la tasa de homicidios aun es un tema para resolver ${ }^{2}$.

La histórica cultura de violencia en Centroamérica obstruye y dificulta el desarrollo económico, político y social de los pueblos, la felicidad y la cooperación individual, familiar, entre comunidades, sociedades y naciones. También dificulta la vivencia de los Derechos Humanos y el desarrollo de la vida misma en condiciones dignas y humanas. Esto conlleva a plantearnos desafíos reales que contribuyan en la superación de la violencia y el delito.

A raíz de esto, es necesario preguntarnos ¿Por dónde empezar a promover la cultura de paz y los derechos humanos? ¿Cómo se pueden materializar diversas iniciativas sobre el tema? ¿Quiénes se beneficiarían directa e indirectamente? ¿Es sostenible la promoción y la implementación curricular en cultura de paz y derechos humanos? ¿qué resultados obtendríamos en la región centroamericana?

\section{Desarrollo}

El marco teórico que fundamenta el presente artículo proporciona de manera general diversos conceptos teóricos - básicos. Teóricamente se encuentra posicionado dentro del campo de la cultura de paz y derechos humanos, los cuales nos acercan a una realidad entendida desde los principios fundamentales que garantizan la vida misma de nuestra especie como homo sapiens.

Si bien, la cultura de paz comprende las situaciones relacionadas una serie de valores, comportamientos y actitudes que rechazan rotundamente cualquier forma de violencia; no podemos dejar de un lado los múltiples acontecimientos que surgen dentro de los sistemas educativos en la región. En el presente escrito, son vistos desde la histórica cultura de violencia que ha tenido la región centroamericana a lo largo de sus casi dos siglos de independencia, y la falta de incorporación y apropiamiento en los currículos educativos de materias específicas tanto en cultura de paz, como derechos humanos.

\footnotetext{
2 Las tazas de homicidio por cada 100,000 habitantes durante el año 2018 en El Salvador son de 51 habitantes, en Honduras es de 40 habitantes, en Guatemala 22 habitantes, Nicaragua (Sin datos), en Costa Rica 11 habitantes, Panamá 9,6 habitantes Recuperado de: https://es.insightcrime.org
} 


\subsection{Derechos humano}

La historia de la humanidad en sus diferentes etapas ${ }^{3}$ ha sido marcada por constantes conflictos y violaciones sistemáticas a la vida y libertad de los individuos. Según Harari (2016) ni en la revolución cognitiva, agrícola, la unificación de la humanidad o en la revolución científica ${ }^{4}$, el homo sapiens no ha podido vivir en paz ni con su misma especie, ni mucho menos con otras especies o medio ambiente. Según Cagliani (2019), en toda la historia de la humanidad, en 5,000 años solo se pudieron vivir 900 años en paz, se han firmado más de 8,00o tratados de paz en los últimos 35 siglos; desde 1945 hasta finales del siglo XX se disputaron 140 guerras con 13 millones de muertes.

El primer principio estipulado en el artículo uno de la declaración fundamental de los derechos humanos plantea que: todos los seres humanos nacen libres e iguales en dignidad y derechos y, dotados como están de razón y conciencia, deben comportarse fraternalmente los unos con los otros. La misma declaración de los derechos humanos, en su artículo tres plantea que: todo individuo tiene derecho a la vida, a la libertad y a la seguridad de su persona (ONU, 1948).

A raíz del segundo holocausto en la historia de la humanidad 5 , el derecho a la vida es el más importante derecho legitimado y legalizado en nuestra sociedad moderna. Sin embargo, la falta de voluntad política de los gobiernos y autoridades regionales y nacionales, han hecho que el derecho a la vida tenga un segundo grado de importancia. En las agendas internacionales comúnmente se hace más eco en el aspecto económico, siendo lo económico el termómetro que mide la calidad de vida de la humanidad.

\subsection{Cultura de paz}

Actualmente, la definición de cultura de paz tiene muchos elementos que deben ser tomados en cuenta. Elementos tales como la promoción de comportamientos y pensamientos no violentos, valores, derechos humanos, costumbres, uso de tecnología, comportamientos individuales y colectivos, la educación, el desarrollo humano, perspectivas incluyentes, la participación política, la información; son elementos que buscan que la cultura de paz se materialice en cada una de las experiencias y en la vida cotidiana de los individuos (Islas, Vera-Hernández \& Miranda-Medina, 2018).

3 Según Marx las etapas de la humanidad se dividen en: Comunismo primitivo, esclavismo, feudalismo, capitalismo, socialismo, comunismo.

4 Yaval Noah Harari divide la historia de la especie humanidad en cuatro partes: La revolución cognitiva, la revolución agrícola, la unificación de la humanidad y la revolución científica. En cada una de estas etapas, el autor plantea que el homo sapiens ha tenido una historia marcada por la violencia entre su misma especie, el medio ambiente, flora y fauna, a tal punto de convertirse en la primera especie exterminadora de su propia vida y de su propio ecosistema.

5 Alonso, A. (2019) Pueblos originarios de la costa caribe nicaragüense. Sobreviviendo a la modernidad social y cultural. Plantea que el primer holocausto en la historia de la humanidad surgió con el contacto con la modernidad colonialista y esclavista de 1492. 
Para Naciones Unidas (1998, Resolución A/52/13), la cultura de paz engloba todo tipo de valores, comportamientos y formas de actuar de cada uno de los individuos que rechazan cualquier forma de violencia; de igual manera, trata de prevenir todo tipo de conflictos desde una cultura preventiva de conflictos. Busca en primer lugar, solucionar los problemas mediante el diálogo y la negociación entre personas, naciones y grupos ${ }^{6}$.

Con el primer foro de cultura de paz celebrado en la región centroamericana, realizado en San Salvador en el año de 1994, se llegó al consenso de que: el objetivo de la Cultura de Paz consiste en lograr que sean los valores de paz los que rijan las soluciones de los conflictos Inherentes a las relaciones humanas (Tünnermann, 1996). En este sentido, la importancia que tienen los diversos sistemas de educación en la región es crucial, ya que al incorporar en su currículo educativo y políticas públicas el tema de cultura de paz, contribuirá enormemente en la transformación social, económica y cultural en la región.

\subsection{El currículo educativo en Centroamérica}

El currículo, técnicamente se refiere a los planes de estudio, planificación anticipada de aprendizaje, programas, implementación didáctica, experiencias de aprendizajes, con el objetivo de producir aprendizajes esperados y planificados (Pérez, 2019). En la actualidad, el currículo tiene la capacidad y el deber de incluir instrumentos esenciales tales como el poder de hablar, discutir y contrastar visiones sobre como concebimos la realidad. También se puede orientar lo que se pretende que en las aulas de clases se enseñe; esto quiere decir que se tiene la posibilidad de orientar al estudiante y a la comunidad educativa hacia caminos de buen vivir bajo una cultura de paz y de derechos humanos (Gimeno, 2010).

Bajo esta premisa, el incorporar en los planes de estudio curriculares los temas de cultura de paz y derechos humanos, permitirá a la región centroamericana contribuir a un cambio de paradigma sin presentes en el continente, sería una apuesta a un futuro inmediato que contribuiría significativamente en los niveles de vida de nuestra población y por consiguiente aportaría enormemente en los temas políticos, económicos y sociales del istmo centroamericano.

Es de resaltar que, si bien desde el año de 1951 con Instauración del comité de cooperación del istmo centroamericano (CCE), las iniciativas integracionistas en la región centroamericana se han centrado en temas meramente económicos y aduaneros

\footnotetext{
6 La resolución identifica ocho ámbitos de acción: Promover la cultura de paz por medio de la educación, el desarrollo económico y social sostenible, el respeto de todos los derechos humanos, Garantizar la igualdad entre mujeres y hombres, 5. Promover la participación democrática, Promover la comprensión, la tolerancia y la solidaridad, Apoyar la comunicación participativa y la libre circulación de información y conocimientos, Promover la paz y la seguridad internacional.
} 
(revise figura 1), quedando a deber los temas de integración curricular y educativos en una de las regiones mas violentas del mundo.

Figura 1: Hitos en la evaluación de la integración econónmica centroamericana

Hitos en la evolución de la integración económica centroamericana, 1951-2018

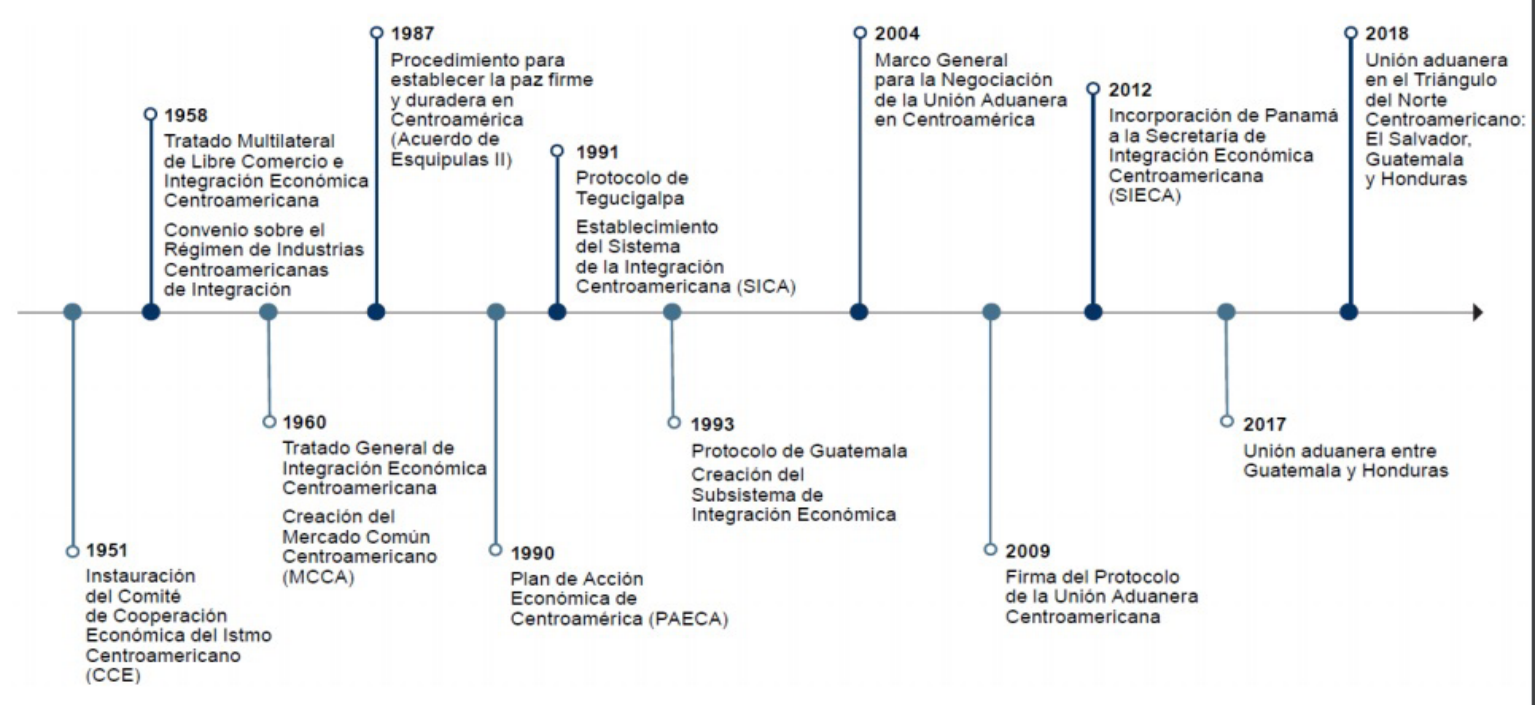

¿Por dónde empezar?

Para llevar a cabo la implementación de una cultura de paz en la región centroamericana, es preciso contar la participación de dos grandes grupos de actores: por un lado, con la voluntad política de los diversos líderes de la región junto con los tres principales sistemas de educación (1. educación preescolar, primera infancia, primaria y secundaria, 2. formación técnica y 3. formación universitaria). Por otro lado, la participación y acción ciudadana de la comunidad educativa, direcciones institucionales, autoridades y actores locales, madres y padres de familia, es imprescindible.

Contando con el apoyo de estos dos conglomerados de actores claves, es indispensable introducir el tema de cultura de Paz en los diversos planes curriculares en los sistemas de educación antes mencionados. Asimismo, seria necesario establecer alianzas con organismos de la sociedad civil, instituciones del estado, autoridades municipales y organismos que trabajen el tema de educación y el tema de cultura de paz, no violencia activa e instituciones y organismos que realicen algún trabajo con la población sensiblemente afectada; esto con el fin de potenciar al máximo la sinergia entre los actores tanto locales como regionales para un mayor impulso de la iniciativa. 


\section{REFLEXIONES EDUCATIVAS}

¿Cómo se materializarían estas primeras acciones en el aula de clase?

Las primeras clases iniciarán con un enfoque claro en la educación en valores humanos como la primera lección del día; esta lección del día, de la semana, del mes y del año será promovida, divulgada y sensibilizada en todas las áreas y por todos los medios creativos en la Comunidad Educativa; en todas partes se dispondrá una expresión de la práctica de los valores humanos; en las relaciones, en los saludos, en la forma de hablar y principalmente en la atmósfera de la escuela, centros técnicos y universidades.

Para este cometido es fundamental el liderazgo comprometido de la dirección de la Escuela, centros técnicos y universitarios junto con docentes promotores y el apoyo de los facilitadores, promotores y voluntarios conformados por la comunidad educativa; estudiantes, maestros, padres y madres de familia y actores claves. Se busca que los estudiantes alcancen un firme progreso hacia el desarrollo de:

- la armonía entre el pensamiento, el sentimiento y la acción;

- una forma de hablar amable y respetuosa hacia sí mismos, así misma, hacia sus propios padre y madres, docentes y compañeros de clases;

- un comportamiento sincero y digno de confianza;

- desarrollar y fortalecer la capacidad de actuar para el mayor bien, su propio bien compatible con el planeta y el bienestar de otros en el presente y en el tiempo.

- un respeto por los sentimientos, valores, creencias, y posesiones de los otros;

- una autoconfianza y buena voluntad de aceptar responsabilidades;

- un entusiasmo de enfrentar sus propios defectos en vez de encontrar fallas en los demás;

- un interés en su trabajo y concentración sostenida;

- un respeto por el medio ambiente, evitando el derroche de los recursos (ej.: energía, tiempo, comida, dinero, agua, etc.);

- un estándar académico de excelencia.

\section{Pertinencia de la Acción}

Las secuelas de la pobreza, el incremento del crimen organizado y el flagelo de las drogas que se cierne amenazante en la región; los gobiernos de Centroamérica han dirigido esfuerzos coordinados para prevenir el crimen organizado, la violencia e inseguridad ciudadana. Sin embargo, los costos del crimen son elevados, tanto en términos económicos asignados en los presupuestos de los países, el costo de seguridad, el deterioro e imposibilidad de generar inversiones, generar trabajo y riqueza y 
en términos de valores por la descomposición social que conlleva la venta y consumo de drogas, el delito y la violencia. La pertinencia de esta iniciativa centroamericana tiene un carácter fundamental en el actual contexto regional, ya que las oportunidades de bienestar y desarrollo sostenible de naciones, familias, grupos, comunidades y personas son potenciadas y sustentadas por la internalización de la cultura de paz en su propia cotidianidad.

\section{¿Quiénes se benefician directamente?}

Los beneficiarios directos los constituyen las familias centroamericanas, junto con su población y las futuras generaciones de estudiantes que se integren los diferentes sistemas de educación regionales. La participación vivencial, activa y conjunta entre los diferentes sistemas educativos centroamericanos, fortalecería las buenas relaciones humanas entre docentes - alumnos - padres y madres de familias y toda la población centroamericana. Es necesario resaltar, que promoviendo los valores de una Cultura de Paz y vivencia de los Derechos Humanos como método de prevención social del delito y la violencia que trasciende en el proceso de socialización a los beneficiaros directos e indirectos en las familias, la comunidad educativa y la población de toda la región centroamericana, con este tipo de iniciativas se podrá realizar una conversión integral de los diversos centros de educación en la región, en centros escolares modelos y exitosos de buenas prácticas inspiradoras para el continente Americano y el mundo como promotores de una cultura de paz.

\section{Beneficiarios Indirectos}

Los beneficiarios indirectos son los siguientes: Personal de las Delegaciones Municipales y Departamentales de los diferentes Ministerios de Educación, centros técnicos y universidades de la región. Centros Escolares, subdirectores, Docentes Educadores, niñez estudiantil, adolescentes y jóvenes, Madres y Padres de familias.

\section{Sostenibilidad de la acción}

Con el fortalecimiento curricular en todos los sistemas educativos de la región, la sostenibilidad de esta acción depende de la actitud individual y colectiva de los países centroamericanos. Por otro lado, la sostenibilidad de esta acción no depende de lo económico sino de la actitud con que se acepte y se lleve a la práctica por los dos grandes conglomerados de actores claves antes mencionados. 
Figura 2: Inversión en educación a nivel centroamericano

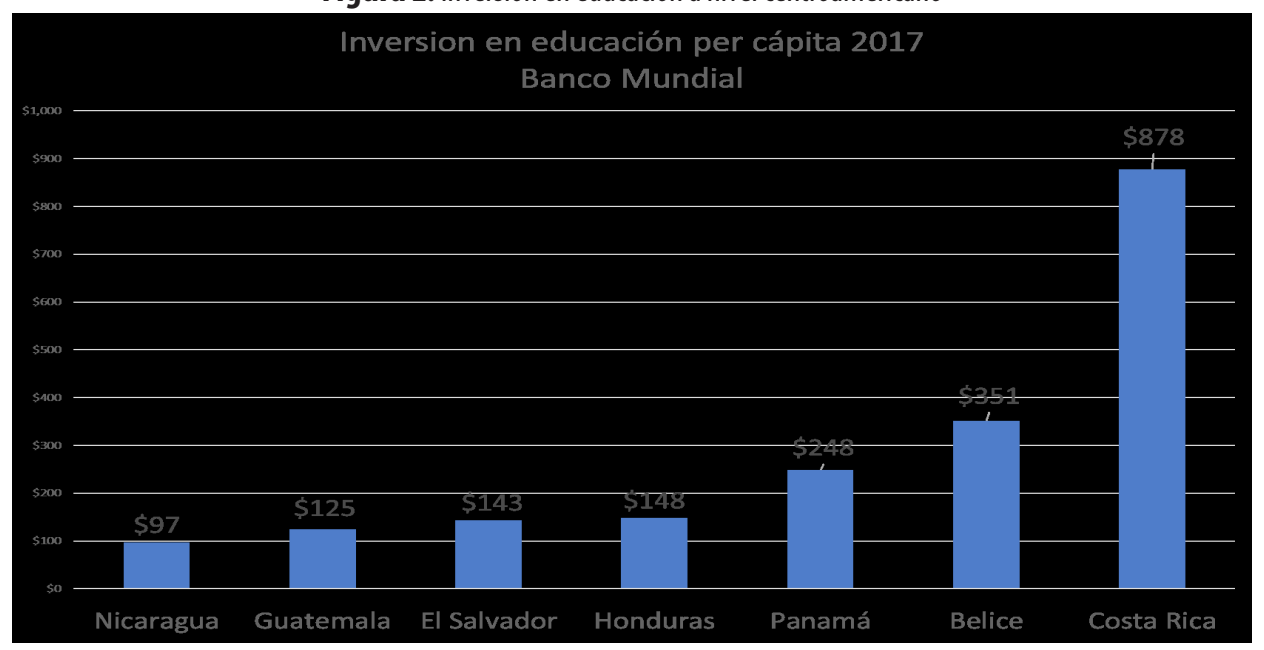

¿Qué resultados podríamos esperar?

Al implementar la acción los resultados esperados son los siguientes:

- Currículo estandarizado en toda Centroamérica en torno a la cultura de paz y derechos humanos en los sistemas de preescolar, primaria y secundaria, educación técnica y universitaria.

- Disminución significativa a largo plazo de los índices de violencia en la región.

- Identificadas las necesidades educativas y formativas sobre cultura y paz y derechos humanos y manifestaciones de violencia más comunes en los tres sistemas educativos antes mencionados.

- Incorporación de los resultados del estudio en la orientación y desarrollo del proyecto a través de la creación de una línea de base que permite medir los resultados e impacto logrados;

- Desarrollada y fortalecida la convivencia pacífica en la Comunidad Educativa;

- Retroalimentado el proceso de empoderamiento de la Comunidad educativa; expresada la vocación de paz y de respeto de los Derechos Humano en la Comunidad Educativa en manifestaciones relacionales, de poder, cotidianas, artísticas y culturales;

- Reflexionadas las causales de la violencia para la sensibilización hacia el cambio de paradigmas, principios, actitudes que coadyuven al desarrollo y promoción de una Cultura de Paz y propósitos de vida compatibles con el planeta donde la meta de felicidad individual incluye a otras personas en el presente y en el tiempo; 
- fortalecida la Red de promotores voluntarios de la Cultura de Paz conformada principalmente por docentes de educación formal, estudiantes, padres y madres de familia, actores locales y líderes comunitarios y religiosos;

- difundidos los valores de una Cultura de Paz para la sensibilización a la población y autoridades de todo el istmo centroamericano.

\section{Conclusiones y perspectivas futuras}

La actual situación de violencia en la región centroamericana es una realidad inminente. La falta de una estandarización curricular que promueva la cultura de paz y derechos humanos en todos los niveles de educación tanto primaria, secundaria, técnica y universitaria, no ha contribuido al mejoramiento en la calidad de vida de la población centroamericana. La región aun presenta los índices de violencia más altos en el continente. La solución de este problema se ha intentado resolver a través del uso de la fuerza y el incremento presupuestario en fuerzas policiales y militares, sin embargo, los resultados aún quedan a deber, resumiéndose en más violencia.

Es por ello, que incorporar la cultura de paz y educación en derechos humanos a los planes de estudio, sería el acto de integración más importante que podemos tener como centroamericanos. Para fortalecer los temas económicos, aduaneros, tecnológicos, comerciales, financieros, energéticos, culturales, sociales y políticos, necesariamente el factor educación y la integración curricular en temas de cultura de paz y derechos humanos, es clave para consolidar las buenas relaciones entre las naciones centroamericanas y su población, traducidas en el buen vivir.

Para ello, es recomendable, que las autoridades competentes en el campo de la educación en sus diversos niveles establezcan una meza conjunta de trabajo curricular, inclusiva, heterogénea, diversa y pluralista. Esto no sería posible, si los gobernantes de cada una de las naciones centroamericanas no lo dimensionan como políticas regionales y como compromisos de estados.

\section{Lista de referencias}

Alonso, A. (2019). Pueblos originarios de la costa caribe nicaragüense. Sobreviviendo a la modernidad social y cultural. Academia de Geografía e Historia de Nicaragua.

Cagliani, A. (2019). De re Militari: Cronología Guerras historia humanidad. Retrieved from http://remilitari.com/cronolog/guerras.htm

Derechos Humanos. (1948). Declaración Universal de los Derechos Humanos, Naciones Unidas. Extraído de: http://www.un.org Fecha de consulta: 17-06-2019 
Fisas, V. (1998). Una cultura de paz. Retrieved from https://escolapau.uab.cat/img/ programas/cultura/una_cpaz.pdf

Gimeno Sacristán, José. (2010). ¿Qué significa el currículum? (adelanto). Sinéctica, (34), 11-43. Recuperado en 28 de junio de 2019, de http://www.scielo.org.mx/scielo. php? script=sci arttext\&pid=S1665-109X2010000100009\&lng=es\&tlng=es.

Harari, Y. (2016). Sapiens de animales a dioses (8th ed.). España: Penguien Random House.

Islas, A., Vera-Hernández, D., \& Miranda-Medina, C. (2018). La cultura de paz en las políticas de Educación Superior de México, Colombia y El Salvador. Revista Educación Y Humanismo, (20/34), 1-15. Retrieved from http://revistas.unisimon. edu.co/index.php/educacion/article/view/2875

Naciones Unidas. (2015). https://www.un.org. Retrieved from https://www.un.org/ es/documents/udhr/UDHR booklet SP web.pdf

ONU. (1998). Retrieved from https://www.um.es/paz/resolucionz.html

Pérez, M. (2019). Conceptos básicos de la teoría curricular. Retrieved from https://www. uaeh.edu.mx/docencia/VI Lectura/LITE/LECT62.pdf

Tünnermann, C. (1996). Cultura de paz. Un nuevo paradigma para Centroamérica (1st ed.). Panamá: Consejo de Rectores. 